\title{
Chlamydiosis of dogs and cats in the modern cities
}

\author{
Vitaliy Nedosekov ${ }^{1,}$, Alexander Martyniuk ${ }^{1}$, Tatyana Stepanova $^{2}$, Valeria Yustyniuk $^{1}$, Irina \\ Gulyukina $^{2}$, Anna Parshikova $^{2}$, and Elena Drozdova ${ }^{2}$ \\ ${ }^{1}$ National University of Life and Environmental Sciences of Ukraine, Kyiv, Ukraine \\ ${ }^{2}$ Federal State Budget Scientific Institution "Federal Scientific Centre (VIEV), 24-1, Ryazanskiy \\ prospekt, 109428, Moscow, Russia
}

\begin{abstract}
The aim of the research was to conduct an in-depth analysis of the investigation of chlamydial infections occurred in cats and dogs in Kyiv during the last 10 years. The article includes three main directions of investigation: 1) The analysis of the epizootic situation with chlamydiosis; 2) Microbiological investigation and 3) Serological monitoring. The clinical and epizootiological studies of chlamydiosis of dogs and cats have been looked into, with the focus on differences and similarities in age and sex of diseased animals. We have investigated 3334 animals (1351 dogs and 1983 cats), including 721 animals (107 dogs and 614 cats) with the manifestation of clinical signs specific to chlamydia. During the investigation 11 isolates of Chlamydia spp. were obtained from the infected animals. Additionally, immune and biological peculiarities have been analyzed. Due to serological monitoring within the epizootiological uncontrolled experiment, a satisfactory level of protection of cats $(63 \%)$ vaccinated against chlamydiosis has been reached, as well as the possibility of the persistence of the pathogen in unvaccinated animals $(9 \%)$.
\end{abstract}

\section{Introduction}

Chlamydia is one of the major infections among animals and birds that have zoonotic origins [1]. Dogs and cats are the most considerable group of domestic animals that inhabit the densely urbanized areas and human residences [2]. Infected animals remain a threat for breeding dogs and cats and may be a potential source of the infectious disease dangerous to people [3, 4].

The analysis of chlamydiosis in Ukraine indicates a lack of data on Chlamydia's species composition, while it circulates and affects dogs and cats in Kyiv. Unfortunately, specialists do not always respond to the manifestation of chlamydiosis in domestic animals on time. They tend to use an outdated devices, storage and data processing systems, which leads to inability to effective control of dogs and cats' chlamydiosis $[3,5]$.

The relevance of the study of these issues is determined by the non-availability of a clear and organized system of epizootiological monitoring of chlamydiosis. The ideal framework would include epizootiological monitoring, the evaluation of risk of Chlamydia infection, a

\footnotetext{
*Corresponding author: nedosekov1@rambler.ru
} 
systematic approach to the identity analysis of the circulating isolates of Chlamydia, and establishment of scientifically based Chlamydia control programs for dogs and cats $[6,7,8]$. Nowadays, monovalent (Katavac Chlamydia ${ }^{\circledR}$, Pfizer, ChlamyCon ${ }^{\circledR}$, Vetzverocenter) and polyvalent inactivated (Multifel-4®, Vetbiochem, Felovax IV®, Fort Dodge Animal Health) vaccines are available for vaccination of cats against chlamydiosis. Although, any vaccine does not guarantee effective protection against the disease due to pathogen peculiarities and administration features. That's why mentioned measures can guarantee a healthy coexistence of domestic animals and society. The purpose of our study was to conduct monitoring and to reassess the epizootiology of dogs' and cats' chlamydiosis in Kyiv (2009-2019).

\section{Materials and methods}

Animals. During 14 years, 3334 domestic animals (1351 dogs and 1983 cats) have been examined. The ambulatory admission record books from Kyiv veterinary clinics have been used for the analysis of the epizootic situation with chlamydiosis of dogs and cats.

In order to gather the microbiological data, the samples taken from 721 animals (107 dogs and 614 cats) with clinical manifestation of suspected chlamydial infection have been looked into. All animals were selected retrospectively on the basis of anamnesis (signs of persistent conjunctivitis, recurrent episodes of ocular discharge, sneezing and nasal discharge, coughing, urogenital syndrome). Information about the animals (clinical signs, age, gender, breed, vaccination status) are shown in Table 1.

Table 1. Age, gender, breed, vaccination status, and clinical signs of cats and dogs suspected of Chlamydia infection.

\begin{tabular}{|c|c|c|}
\hline Parameters & Cats & Dogs \\
\hline Age & $\begin{array}{l}5 \text { week }-9 \\
\text { years old }\end{array}$ & $\begin{array}{l}3 \text { months }-9 \text { years } \\
\text { old }\end{array}$ \\
\hline \multicolumn{3}{|l|}{ Sex } \\
\hline Male & 359 & 57 \\
\hline Female & 255 & 50 \\
\hline \multicolumn{3}{|l|}{ Breed: } \\
\hline Persian & 28 & - \\
\hline Scottish fold & 29 & - \\
\hline Siamese & 37 & - \\
\hline Maine coon & 31 & - \\
\hline British shorthair & 44 & - \\
\hline Himalaya cat & 22 & - \\
\hline Mixed breed & 423 & 23 \\
\hline Rottweiler & - & 11 \\
\hline American staffordshire terrier & - & 17 \\
\hline Pekingese & - & 18 \\
\hline Japanese Chin & - & 10 \\
\hline German shepherd & - & 15 \\
\hline French bulldog & - & 13 \\
\hline \multicolumn{3}{|l|}{ Vaccination status } \\
\hline Multifel-4® & 5 & - \\
\hline Katavac Chlamydia ${ }^{\circledR}$ & 7 & - \\
\hline ChlamiCon $\AA$ & 5 & - \\
\hline Felovax-IV® & 2 & - \\
\hline Non-vaccined & 35 & - \\
\hline Unknown vaccination status & 424 & - \\
\hline Clinical signs: & & \\
\hline
\end{tabular}




\begin{tabular}{|l|c|c|}
\hline $\begin{array}{l}\text { persistent conjunctivitis, ocular discharge, } \\
\text { blepharospasm }\end{array}$ & 436 & 24 \\
\hline $\begin{array}{l}\text { Eye lesions and respiratory system lesions } \\
\text { (sneezing and nasal discharge, rhinitis) } \\
\text { coughing }\end{array}$ & 135 & - \\
\hline Eye lesions and urogenital tract lesions & - & 54 \\
\hline $\begin{array}{l}\text { urogenital system lesions (including } \\
\text { vaginitis, balanoposthitis, infertility, } \\
\text { abortions and asymptomatic course) }\end{array}$ & 43 & 29 \\
\hline Total number of examined animals & 614 & 107 \\
\hline
\end{tabular}

Samples. During 14 years a total of 967 samples from cats and 161 samples from dogs were examined (Table 2). All samples were taken correspondingly to the clinical manifestation of the disease. If it was necessary, different types of samples were taken from the same individual (for example, in the case of eye lesions and respiratory system lesions, samples were taken both from conjunctiva and pharynx or nasal cavity).

A total of 526 paired conjunctival swab samples were obtained from the ventral conjunctival fornix, using sterile cotton-topped swabs.

A total of 423 nasal swabs were obtained from the anterior nostril by sweeping upwards towards the top of the nostril. Sterile cotton-topped swabs were used.

A total of 53 pharyngeal swabs were obtained from the lateral wall of pharynx, using sterile cotton-topped swabs.

A total of 85 vaginal swabs were obtained by passing a cotton-topped swab into a caudal vagina. The swab was directed craniodorsal when entering the vaginal vault in order to avoid the clitoral fossa.

A total of 18 urethral swab specimens were collected by inserting the specimen collection swab 2 to $4 \mathrm{~cm}$ into the urethra, rotating the swab clockwise for 2 to 3 seconds to ensure adequate sampling.

All swabs were collected without local analgesia. All procedures on animals were reviewed by a local animal care committee to ensure that the procedures were appropriate and humane. The acquisition, care, housing, use, and disposition of animals in research was in compliance with applicable state and local laws and regulations, institutional policies, and with international conventions.

Table 2. Origin of the investigated samples.

\begin{tabular}{|l|c|c|}
\hline \multicolumn{1}{|c|}{ Samples } & Cats & Dogs \\
\hline Conjunctival swabs & 448 & 78 \\
\hline Nasal swabs & 423 & - \\
\hline Pharyngeal swabs & 53 & - \\
\hline Urogenital swabs: & & \\
\hline \multicolumn{1}{|c|}{ vaginal swabs } & 31 & 54 \\
\hline urethral swabs & 12 & 29 \\
\hline Total & 967 & 161 \\
\hline
\end{tabular}

Microbiological investigation. Microbiological culture was performed in the Laboratory of Microbiology and Virology of Institute of Veterinary Medicine NAAS of Ukraine (IVM) accordingly to the common used methods.

The growth of the gram-positive and gram-negative microorganisms (Staphylococcus spp., Streptococcus spp., Diplococcus spp., Micrococcus spp., Sarcina spp., Pseudomonas spp., Escherichia spp.) was determine on the meat peptone agar and liver infusion agar medium at the temperature of $37^{\circ} \mathrm{C}$ for up to 10 days.

The growth of the microorganisms of the genus Mycoplasma was determined by cell culture on the complex Edward medium ( $20 \%$ horse serum, $10 \%$ yeast extract, $0.5 \%$ glucose 
and phenol red $0.001 \% \mathrm{w} / \mathrm{v}$ aqueous stock solution) at the temperature of $37^{\circ} \mathrm{C}$ for up to 21 days.

Polymerase chain reaction (PCR) was conducted in the "Center for diagnosis of animal diseases" (LLC "Bald"). All DNA samples were tested by using the PCR procedure described by Ksonz I. (2013). Optimal condition of PCR:- 2,5 mkl 10- multiple buffer (670 MM Tris c-HCI, pH 8,8 t $-25^{\circ} \mathrm{C}, 20 \mathrm{mM}$ bovine albumin, $166 \mathrm{mM}\left(\mathrm{NH}_{4}\right)_{2} \mathrm{SO}_{4}, 100 \mathrm{mM} \mathrm{2- \beta -}$ merkaptoetenol), $1 \mathrm{mcl} 2,5 \mathrm{mM}$ dNTP, $0,5 \mathrm{mcl}$ of each of primers, $1,5 \mathrm{mcl} 50 \mathrm{mM} \mathrm{MgCl}_{2}$, specimen DNA and $1 \mathrm{mcg} / \mathrm{ml}, 2-3$ UI. Taq DNA-polymerase [15]. The primers targeted conservative $16 \mathrm{~S}$ ribosomal RNA gene of the bacterial family Chlamydiaceae. The oligonucleotides were synthesized by Liteh (Russia).

The biological properties of Chlamydia spp. (infectious, immunogenic, cultural and pathogenic) were examined using 11 isolates (CAT-1, CAT-2, CAT-3, CAT-4, CAT-5, CAT-6, CAT-7, CAT-8 and D-1, D-2 and D-3) of Chlamydia, obtained from cats and dogs with chlamydiosis confirmed by PCR. Chlamydia isolate CAT-1(7.0-7.3 $\left.1 \mathrm{gLLD}_{50} / \mathrm{cm}^{3}\right)$ was used for the development of immunity reagents. That type of isolate was received in 2009 from conjunctival swabs of cats and identified in the Complement-Fixation Test (CFT) with specific Chlamydial serum (Federal Centre for Toxicological, Radiation, and Biological Safety, Russian Federation) and determined in the polymerase chain reaction (PCR) as a type of Chl. felis [4].

The titer of Chlamydia was determined by titration on chicken embryos obtained from a specific pathogen free flocks. Chlamydia titer was calculated with the help of the Kerbers method and expressed in $\mathrm{ELD}_{50} / 0.3 \mathrm{~cm}^{3}$.

Specific destruction of embryos was verified by immunofluorescence test (IF), conducted with the usage of a diagnostic kit "Hlamonoskryn" and fluorescent chlamydial monoclonal antibody, with the serial number 24-121106 (LLC "Niarmedic-Plus", Russian Federation).

To control Immunofluorescence, positive and negative patterns (series 14-220607), (LLC "Niarmedic-Plus", Russian Federation) have been used. The frequency of experiments was $\geq 3$, which ensured the reliability of the results. The numerical data was exposed to statistical analysis conducted by in MS Excel on a PC Intel and application package "Statistics" (version 5.0).

To conduct the study of immunogenic and reproductive properties of Chlamydia isolates, the identification of the pathogen in the yolk membrane of chicken embryos was done successful in 28 cases, 17 of which had been contaminated by extraneous microorganisms. For further research 11 isolates of Chlamydia, obtained from chicken embryos free from extraneous microorganisms were selected. The first phase had a selection of isolates of Chlamydia in terms of immunogenicity and reproduction. For this purpose, eleven chicken embryos had been inoculated with the selected isolates of Chlamydia into yolk membrane. The material containing Chlamydia from each isolate with infectious activity of not less than 2,0 $\mathrm{lg} \mathrm{ELD}_{50} / \mathrm{cm}^{3}$ was inactivated and injected into three guinea pigs (intraperitoneally and intrathoracially, in the volume of $1 \mathrm{~cm}^{3}$ per animal).

Serological monitoring of Chlamydiosis was conducted within the epizootic, uncontrolled experiment, which aim was to identify the immune status of cats regardless of the multiplicity of vaccination and time after immunization. In terms of uncontrolled experiment two groups of animals were tested (vaccinated - 19, and not vaccinated - 35) for the presence of specific antibodies to Chlamydia pathogen by CFT in triplicate.

Through CFT, 54 samples of blood serum obtained from cats in Veterinary Clinics were studied. 


\section{Results}

During our research, three main directions of epizootiological monitoring have been determined:

i) Analysis of the epizootic situation with chlamydiosis of dogs and cats in Kyiv;

ii) Microbiological investigation;

iii) Serological monitoring.

\subsection{Analysis of the epizootic situation with chlamydiosis}

Within 10 years, 3334 animals (1351 dogs and 1983 cats) were examined, among which 721 animals (107 dogs and 614 cats) had clinical manifestation of suspected chlamydial infection and have been identified as potential chlamydiosis carriers. However, chlamydiosis was confirmed by PCR in 341 animals only (46 dogs and 295 cats) out of 721 animals. It was found that $47.3 \%$ of the total number of animals with Chlamydial infection had eyes lesions and lesions in respiratory and urogenital systems, which correlated with the symptoms of the disease. Thus, it was confirmed that the number of chlamydiosis cases among dogs and cats in Kyiv had the following frequency: $3.4 \%$ dogs (46 animals), $14.9 \%$ cats (295 animals).

In addition to Chlamydia spp., Sarcina conjunctivae, Pseudomonas aeruginosa and Escherichia coli were also indicated in dogs and cats with the symptoms of conjunctivitis such as reddening of the eyes. In the nasal and pharyngeal swabs from cats with the signs of rhinitis, Staphylococcus pharyngis, Diplococcus lancelatus and Microccocus saccatus were found.

Pets with balanoposthitis also carried Staphylococcus alba, Staphylococcus aureus, Staphylococcus citreus, Bact. pyocyaneum, Micrococcus ureae, Proteus ammoniae, Candida albicans, and even Hemophilus canis, with vaginitis - Staphylococcus alba, Staphylococcus aureus, Streptococcus felines, Micrococcus minimus, Trichomonas vaginalis, Gardnerella vaginalis, E. coli, Proteus vulgaris and Candida albicans.

About $27 \%$ of chlamydiosis cases were recorded in association with Mycoplasma spp., which was the most commonly associated infectious agent. Therefore, chlamydial infection, either mixed or secondary, occurred in association with other microorganisms and revealed through a wide range of clinical signs.

Analysis with respect to age and breed of cats and dogs with chlamydial infection in Kyiv showed that among the studied cats the largest number of registered patients was between 5 weeks and 3 years $(70 \%)$. Kittens under 5 weeks, and cats of 3 years and over were less vulnerable to the disease, $12 \%$ and $18 \%$ respectively. Susceptibility to infection with Chlamydia was observed in dogs aged from 2 months to 3 years and over 6 years $(59 \%)$. Animals under one year generally had chlamydial conjunctivitis, whereas older ones (in addition to conjunctivitis) showed vaginitis, balanoposthitis, infertility and abortions.

A possible connection between the breeds and animals' predisposition to the chlamydiosis disease was not established. However, we have managed to differentiate chlamydia between Staffordshire terrier (6), Pekingese (7), Japanese Chin (4), Rottweiler (5), German shepherd (6), French bulldog (3) and unbreed dogs (15). The most vulnerable to chlamydiosis among cats were such breeds as Persian and British shorthair. Thus, the symptoms of chlamydiosis in pets included conjunctivitis with frequent lesions of respiratory (rhinitis, tracheitis) and urogenital systems.

The analysis of the clinical and epizootiologic features of chlamydiosis in dogs and cats. It was found that $48 \%$ of all cats (295 animals) with diagnosis of chlamydial infection confirmed by PCR had conjunctivitis with symptoms of nictitating spasm (spasm of the eyelid with continuous winking) and chemosis, and complications of the respiratory and urogenital systems lesions (Fig.1). 


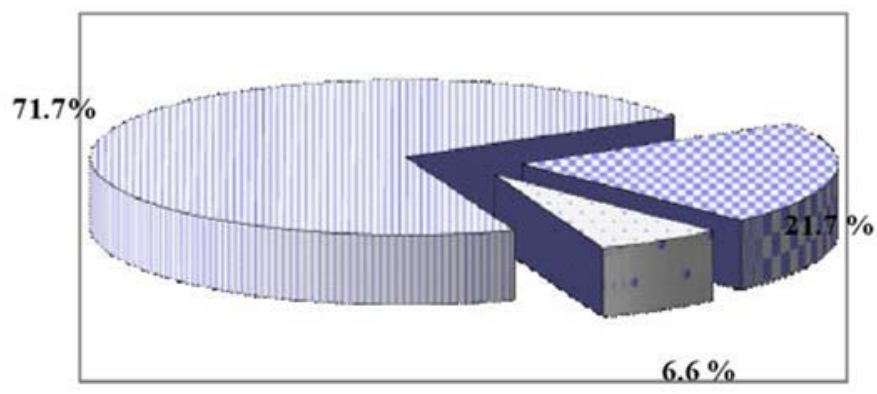

\section{口ophthalmological syndrome \\ 口ophthalmological syndrome with lesions in respiratory system \\ 口ophthalmological syndrome with lesions in urogenital system}

Fig. 1. The structure of the clinical manifestations of chlamydiosis in cats.

Consequently, in cats the disease clinically manifested as ophthalmological syndrome in $71.7 \%$ (211 animals) of cases, complicated by lesions in respiratory system (rhinitis, tracheitis, pneumonia) in $21.7 \%$ (64 animals), and urogenital system in $6.6 \%$ (20 animals).

Figure 2 represents main clinical manifestation of chlamydiosis in dogs.

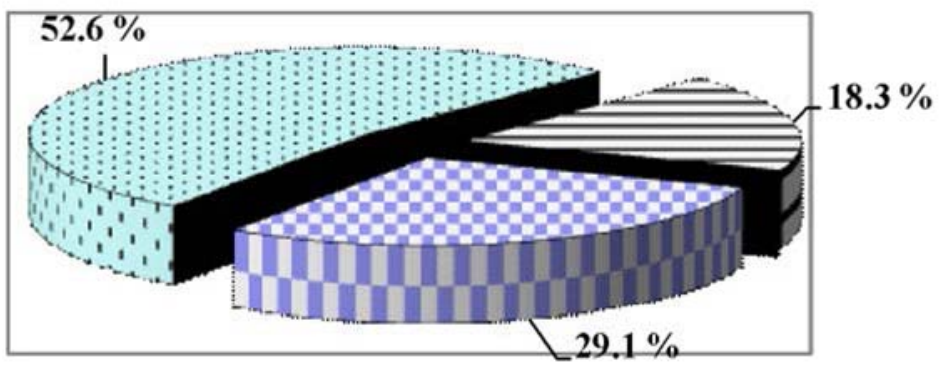

\section{घophthalmologicalsyndrome with lesions in urogenital system}

\section{口ophthalmologicalsyndrome}

\section{口lesions in urogenital system}

Fig. 2. The structure of the clinical manifestations of chlamydiosis in dogs.

As follows, chlamydiosis in dogs was characterized by ophthalmological syndrome in $52.6 \%$ cases (25 animals), lesions in urogenital system in $18.3 \%$ (8 animals) or both problems simultaneously in $29.1 \%$ cases (13 animals).

\subsection{Microbiological monitoring of Chlamydiosis}

The monitoring was carried out by examining of 1128 samples from 721 animals suspected of being infected and infected with chlamydiosis. According to the results of PCR, chlamydiosis was found in 295 samples from cats and 46 from dogs, however, identification of the pathogen in the yolk membrane of chicken embryos was successful only in 28 cases, 
17 of which had been contaminated by extraneous microorganisms. Conducting microbiological monitoring, 11 isolates free of extraneous microorganisms were selected for further research.

The results of the study of immunogenic and reproductive Chlamydia isolates properties are presented in Table 3.

Table 3. Evaluation of immunological properties of Chlamydia isolates, from dogs and cats.

\begin{tabular}{|c|c|c|c|c|}
\hline № & $\begin{array}{c}\text { Isolates of } \\
\text { Chlamydia }\end{array}$ & $\begin{array}{c}\text { Infectious activity of } \\
\text { chlamydia in the yolk } \\
\text { membrane of chicken } \\
\text { embryos * }\end{array}$ & $\begin{array}{c}\text { Days of } \\
\text { embryos death } \\
\text { after } \\
\text { inoculation }\end{array}$ & $\begin{array}{c}\text { The titer of antibodies } \\
\text { in the serum of guinea } \\
\text { pigs ** }\end{array}$ \\
\hline 1 & CAT-1 & $3.2^{ \pm} 0.2$ & $7-9$ & $1: 80$ \\
\hline 2 & CAT-2 & $2.3^{ \pm} 0.4$ & $8-9$ & $1: 20$ \\
\hline 3 & CAT-3 & $2.3^{ \pm} 0.2$ & $8-9$ & $1: 10$ \\
\hline 4 & CAT-4 & $2.6^{ \pm} 0.2$ & $8-9$ & - \\
\hline 5 & D-1 & $2.0^{ \pm} 0.3$ & $10-11$ & $1: 20$ \\
\hline 6 & CAT-5 & $2.8^{ \pm} 0.2$ & $8-10$ & $1: 80$ \\
\hline 7 & D-2 & $2.4^{ \pm} 0.3$ & $10-11$ & - \\
\hline 8 & CAT-6 & $2.2^{ \pm} 0.2$ & $8-10$ & $1: 10$ \\
\hline 9 & CAT-7 & $3.0^{ \pm} 0.2$ & $8-9$ & - \\
\hline 10 & CAT- 8 & $2.6^{ \pm} 0.2$ & $7-9$ & $1: 40$ \\
\hline 11 & D-3 & $2.5^{ \pm} 0.3$ & $10-12$ & \\
\hline
\end{tabular}

* Infectious activity in Ig $\mathrm{ELD}_{50} / \mathrm{cm}^{3}$;

** Average sample from three animals.

At the highest titer, isolate CAT-1 $\left(3,2 \mathrm{lg} \mathrm{ELD}_{50} / \mathrm{cm}^{3}\right)$ was accumulated at the level of anti-chlamydia antibodies 1:80. Isolate CAT-1 appeared to be the most suitable for the development of technology for immune reagents to diagnose chlamydiosis and study biological properties. This type was determined by PCR and it established its belonging to the Chlamydophila felis species; order - Chlamydiales; family - Chlamydiaceae; genus Chlamydophila

\subsection{Serological monitoring of Chlamydiosis}

According to the recommendations of the vaccine products, antibody level which protects against infection of chlamydia should be not less than 1:8-1:16 [3]. Results of CFT of 54 cats' blood serum are presented in Table 2.

Table 2. The results of blood serum testing in cats.

\begin{tabular}{|c|c|c|c|c|}
\hline \multirow{2}{*}{$\begin{array}{l}\text { № } \\
\text { s/n }\end{array}$} & \multirow{2}{*}{$\begin{array}{l}\text { Name of } \\
\text { vaccines }\end{array}$} & \multirow{2}{*}{$\begin{array}{c}\text { The } \\
\text { number of } \\
\text { animals } \\
\text { examined }\end{array}$} & \multicolumn{2}{|c|}{$\begin{array}{l}\text { The number of samples with antibody titers } 1: 8 \text { and } \\
\text { higher }\end{array}$} \\
\hline & & & Animals & Percentage \\
\hline 1 & Multifel4® & 5 & 3 & 60 \\
\hline 2 & $\begin{array}{l}\text { Katavac } \\
\text { Chlamydiaß }\end{array}$ & 7 & 6 & 85.7 \\
\hline 3 & ChlamiCon ${ }^{\circledR}$ & 5 & 2 & 40 \\
\hline 4 & Felovax-4® & 2 & 1 & 50 \\
\hline 5 & Non-vaccined & 35 & 3 & 8.6 \\
\hline 6 & Total & 54 & 15 & 27.8 \\
\hline
\end{tabular}

As follows, 12 of 19 vaccinated animals $(63.2 \%)$ had the protective antibody level. 
However, 3 of 35 unvaccinated animals showed the presence of anti-chlamydia antibodies in the titer of 1:8 - 1:64, which may suggest the persistance of Chlamydial pathogen in the time of testing (no clinical manifestation was observed).

\section{Discussion}

According to numerous reports, chlamydiosis is a widespread disease among cats in the world, particularly in Japan, where it is registered in $10 \%$ of cats. Among cats a prevalence of $35 \%$ was detected in Canada, $65 \%$ in Germany, $49 \%$ in France, $25 \%$ in Russia; in Switzerland it's $48 \%$. In UK it's $21 \%$ and in the USA it's $47 \%$.

The results of this study demonstrated, that cat's chlamydiosis with ophthalmological syndrome occurs in $71.7 \%$ of animals with clinical manifestation. We believe that was due to the presence of unsupervised pathogen's reservoir in the nature [5]. To confirm this, we have investigated 3334 animals (1351 dogs and 1983 cats), including 721 animals (107 dogs and 614 cats) with clinical manifestation of suspected chlamydial infection. However, the diagnosis of chlamydiosis was confirmed by PCR in 341 cases only. Of the total number of examined animals with ophthalmological syndrome and signs of lesions of respiratory and urogenital systems, Chlamydia was present in $47.3 \%$ cases, confirming the leading role of chlamydiosis in animals with those symptoms in Kyiv region.

Also, the study of our colleagues showed that the clinics of Veterinary Medicine in Wroclaw annually treat 600 cats; among which $50 \%$ have ophthalmological problems, and $30 \%$ - are diagnosed with chronic conjunctivitis. Chlamydophila felis is the main cause of chronic conjunctivitis in cats, indicating that the pathogen is spread among cats $[9,10]$. In Europe, detection of Chlamydia DNA ranged from $3.3 \%(2 / 60)$ in healthy cats to $20 \%$ $(14 / 70)$ of animals with conjunctivitis in Italy [11]. The percentage of positive animals (Chlamydophila felis) was $14.7 \%$ in UK [12], $11 \%$ in Switzerland [13], and $15.3 \%$ in Sweden [3]. In Japan, while studying the DNA of Chlamydophila, the scientist found $59.1 \%$ positive results, and the pathogen was identified in 11 of the 39 cats that reacted positively in the PCR [14].

The peculiarity is that the disease can occur in cats with clinical signs and in healthy ones (asymptomatic carriers); although Rampazzo et al say that presence of Chlamydophila correlates with conjunctivitis [11]. According to our research, the disease of cats is accompanied by an ophthalmological syndrome in $71.7 \%$ of cases, by complicated lesions of the respiratory (rhinitis, tracheitis, pneumonia) in $21.7 \%$ and by urogenital system in $6.6 \%$.

It should be noted that there is no literature data regarding clinical manifestations of chlamydiosis in dogs. However, Ksonz et al. established that the causative agent of the disease in dogs are often Chlamydia pecorum, Chlamydia psittaci or Chlamydia abortus. These are the Chlamydia species that were identified while differentiating samples collected from dogs with chlamydiosis [15].

The most common form of chlamydiosis in dogs is genital. Lesions of urogenital tract and reproduction pathology were registered in $67 \%$ of cases. This form was established by infertility, hidden and symptomatic abortion, stillborn and poor vital pups $[15,16,17,18$, 19].

Our results are somewhat different: in dogs chlamydiosis is characterized mainly by ophthalmological syndrome $(52.6 \%)$, by lesions in urogenital system $(18.3 \%)$, or by both problems simultaneously ( $29.1 \%$ of cases). Moreover, among cats and dogs chlamydiosis did not run as a monoinfection. Thus, $39.3 \%$ of cases with confirmed chlamydial infection in cats were recorded in association with mycoplasmas, which were the most common infectious agent that accompanied chlamydiosis [20]. 


\section{Conclusion}

The results of this investigation show the prevalence of Chlamydial infection in cats and dogs in Kyiv, which is a huge problem for breeding dogs and cats and a reason for public health concern because both dogs and cats could be reservoir hosts of Chlamydia in humans. To our knowledge, this is the first report of chlamydiosis of cats and dogs in Kyiv. It also shows that the data concerning chlamydiosis of pets are still fragmentary.

\section{References}

1. M. Krawiec, T. Piasecki, A.K. Wieliczko, Prevalence of Chlamydia psittaci and Other Chlamydia Species in Wild Birds in Poland Vector-borne and zoonotic diseases 15(11) (2015)

2. A.K. Wieliczko, K. Płoneczka-Janeczko, Pol. J. Vet. Sci. 13(2), 381-3 (2010)

3. B. Holst Ström, L. Englund, S. Palacios, L. Renström, L.T. Berndtsson, J. Feline. Med. Surg. 8, 207-211 (2006)

4. O.G. Martyniuk, The epizootological monitoring and the improvement of the canine and feline chlamydiosis diagnostic means. Dissertation for obtaining of veterinary sciences on the specialization 16.00.03 (The National University of Life and Envionmental Sciences of Ukraine, Kyiv, 2011)

5. V.V. Nedosiekov, A.G. Martynyuk, I.M. Ksonz, T.N. Tsivenko, Veterinary Medicine of Ukraine 6, 10-12 (2010)

6. Wu et al., BMC Veterinary Research 9, 104 (2013)

7. K. Rypula, A. Kumala, P. Lis, K. Niemczuk, K. Płoneczka-Janeczko, Z. Pejsak, Pol. J. Vet. Sci. 17(2), 367-9 (2014)

8. C. Schulz, K. Hartmann, R.S. Mueller, C. Helps, B.S. Schulz, J. Feline Med. Surg. 17(12), 1012-9 (2015) doi: 10.1177/1098612X15569615

9. A.M. Gulyukin, V.V. Belimenko, A.A. Shabeykin, A.E. Droshnev, A.I. Laishevtsev, IOP Conference Series: Earth and Environmental Science 421, 042013 (2020)

10. V.V. Belimenko, P.I. Khristianovsky, E.V. Novosad, A.M. Gulyukin, IOP Conf. Ser.: Earth Environ. Sci. 315, $042024 \quad$ (2019) https://doi.org/10.1088/1755$1315 / 315 / 4 / 042024$

11. V.V. Belimenko, A.M. Gulyukin, IOP Conference Series: Earth and Environmental Science 548, 42039 (2020)

12. M. McDonald, B.J. Willett, O. Jarrett, D.D. Addie, Vet. Rec. 143, 97-101 (1998)

13. W. Von Bomhard, A. Polkinghorne, Z.H. Lu, L. Vaughan, A. Vogtlin, D.R. Zimmermann, B. Spiess, A. Pospischil, Am. J. Vet. Res. 64, 1421-1428 (2003)

14. Y. Cai, H. Fukushi, S. Koyasu, E. Kuroda, T. Yamaguchi, K. Hirai, J. Med. Vet. Sci. 64, 215-219 (2002)

15. I. Ksonz, Chlamidiosis of animals (epizootology, etiology, diagnosis, sanation). Dissertation for obtaining of veterinary sciences on the specialization 16.00.03 (The National University of Life and Envionmental Sciences of Ukraine, Kyiv, 2013)

16. V. Nedosekov, International scientific electronic journal Earth Bioresources and Quality of Life 1 (2012) http://gchera-journal.nubip.edu.ua/index.php/ebql/article/view/14

17. M. Bannasch, J. Foley, J. Feline Med. Surg. 7, 109-119 (2005) 
18. K. Płoneczka-Janeczko, Z. Kiełbowicz, M. Kuczkowski, Poland Vet. Inst. Pulawy. 55, 193-198 (2011)

19. A. Rampazzo, S. Appino, P. Pregel, A. Tarducci, E. Zini, B. Biolatti, J. Vet. Int. Med. 17, 799-807 (2003)

20. A.M. Gulyukin, Voprosy virusologii 59(3), 5-10 (2014) 